\title{
THE CALCULATIONS OF AREAS AND VOLUMES USING THE METHOD OF ARCHIMEDES: SOME DIDACTIC CONSIDERATIONS
}

\author{
Paolo Bussotti \\ University of Udine, Italy
}

An interdisciplinary approach to education is nowadays considered an important aspect to improve the critical skills of the learners so that they can guess how several aspects of the human knowledge are interconnected. A key aspect of interdisciplinary education is represented by the use of the history of a certain subject within the teaching of the subject itself. This is particularly conspicuous in science education. For, the appropriate and not superficial introduction of historical elements within science education allows the pupils to discover the human aspects of science, the problems behind the creation and development of many concepts, which are often presented only in a formal manner. As a matter of fact, after having grasped that some problems are difficult and after having understood and appreciated the efforts that in the course of history the scientists have carried out to solve such problems, the learners will accept the necessity of a formalization and will not consider such a formalization as a sort of abstract doctrine imposed by the teachers for unspecified reasons. Furthermore, history of science has profound relations with history, mathematics, science, philosophy, physique and technique, so that it is a typical interdisciplinary subject which can be exploited in an educative context.

The considerations which follow concern the calculations of areas and volumes of curvilinear and mixtilinear figures. At a first glance, one might think that the problem is connected only to mathematics, to its history and its education, but, in fact, this is not the case, because it encompasses the whole of exact sciences education insofar as it is related to the concept of integral, which is one the mathematical basic notions used in physics and in all the mathematized sections of other sciences.

In general integral calculus is taught at the last but one or the last year of scientific or technical gymnasia/ high schools. In most cases the concept of definite integral is introduced in the form of Riemann integral or Cauchy integral. In both cases an enough heavy formal apparatus is necessary. Obviously the relation between the concept of definite integral and the calculations of plane areas is explained and the fundamental theorem of integral calculus is proved. The whole of this approach is correct, but is there a more intuitive way which allows the learners to understand why the resort to a formal apparatus is appropriate and which, at the same time, make them guess the background of problems which induced the mathematicians to work on the precise definition of the concept of integral? This way exists and it passes through history of mathematics.

The first and fundamental phase of our proposed itinerary which connects mathematics and science education with mathematics and science history takes place in Siracusa, the Sicilian city where in the third century B.C. the greatest mathematician of antiquity and one of the greatest ever worked: Archimedes.

Euclid, some decades before Archimedes, had written his Elements, the fundamental text of Greek geometry, where, in part collecting a series of knowledge already existing in Greek mathematics, in part adding his new results, had offered an impressive picture of Greek geometry. The width of the subject dealt with by Euclid as well as his rigorous approach made the Elements an unavoidable reference point for all the mathematicians active after Euclid. The problem of the areas of rectilinear flat figures is faced in the first and, after the introduction of the concept of proportion (fifth book), in the sixth book of the Elements. Euclid is, in substance, able to solve the problem reducing the calculation of rectilinear flat areas to the calculation of the areas of triangles. However, with regard to curvilinear areas and volumes, things are by far more difficult. All the results obtained by Euclid are referred to in the $12^{\text {th }}$ book: in proposition 2, Euclid proved that the circles are as the squares of their diameters; in proposition 10 that the volume of a cone is equal to $1 / 3$ of that of a cylinder having its same base and height; in proposition 11 that cones and cylinders having the same heights are as the bases; in proposition 12 that similar cones and cylinders are as the cubes of their bases' diameters; finally, in proposition 18 that the volume of a sphere is proportional to the cube of its 
diameter. In all the proofs of these propositions Euclid used a particular demonstrative method, of which probably Eudoxus was the inventor: the method of exhaustion.

The first question to pose from a conceptual and didactical standpoint is the following: can the exhaustion method be considered a key to calculate the areas and the volumes of curvilinear and mixtilinear figures? The answer to this question cannot be found in Euclid, but in Archimedes because he extended the exhaustion method by far beyond the limits within which it was used by Euclid. We will see that the logical and conceptual nuances of this method are in themselves an amazingly interesting subject for science and mathematics education if it is true that mathematics is not only the learning of a series of techniques and results, but also a training to refine our mind, logic and reasoning capabilities. The impressive results obtained by Archimedes through exhaustion are summarised in this table:

\begin{tabular}{|c|c|}
\hline Work & Main results through exhaustion \\
\hline Measurement of a Circle & Area of a circle \\
\hline \multirow[t]{6}{*}{ On the Sphere and the Cylinder (two books) } & 1. Lateral surface of a right cylinder $(I, 13)$. \\
\hline & 2. Lateral surface of an isosceles cone $(I, 14)$. \\
\hline & 3. Surface of a sphere $(I, 33)$ \\
\hline & 4. Volume of a sphere $(1,34)$ \\
\hline & 5. Surface of spherical segments $(I, 42,43)$. \\
\hline & 6. Volume of a spherical sector $(I, 44)$. \\
\hline \multirow[t]{2}{*}{ The Quadrature of the Parabola } & 1. Area of a parabolic segment ( 16 , first proof). \\
\hline & 2. Area of a parabolic segment ( 24 , second proof). \\
\hline \multirow[t]{4}{*}{ On Conoids and Spheroids } & 1. Area of the ellipsis (4). \\
\hline & 2. Volume of the paraboloid of revolution $(21,22)$. \\
\hline & 3. Volume of the hyperboloid of revolution $(25,26)$. \\
\hline & 4. Volume of the ellipsoid of revolution $(27,28)$. \\
\hline \multirow[t]{5}{*}{ On Spirals } & 1. Circumference of the "first circle" of the spiral (18). \\
\hline & 2. Circumference of the "second circle" of the spiral (19). \\
\hline & 3. Perimeter of arches of circles of the spiral (20). \\
\hline & 4. Surface of the spiral described in the first and second rotation (24 and 25 resp). \\
\hline & 5. Surface of an arch of spiral (26). \\
\hline
\end{tabular}

To develop our discussion, I will explain the easiest application of exhaustion method developed by Archimedes: the calculation of the circle's area. Archimedes claimed:

Any circle $A B C D$ is equal to the right triangle $E$ of which the radius is a small side and the circumference is the other small side.
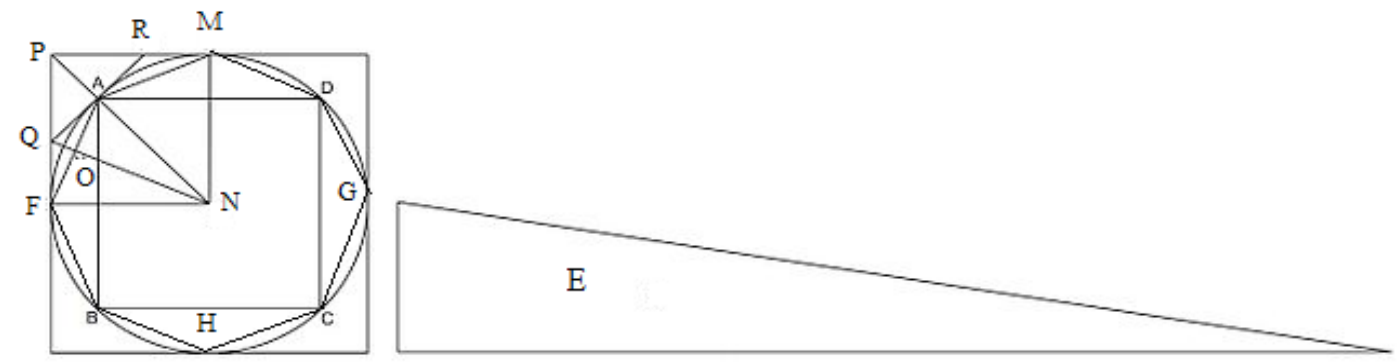

Figure 1. The diagram used by Archimedes to find the area of the circle.

Proof: suppose that the area of $A B C D$ is bigger than $E$. It is possible to inscribe the square $A C$ within the circle $A B C D$. Bisect the four arches of circumference so obtained (in Figure 1 the divisions points are indicated by $F, M, G$, $H$ ). Eight circular segments congruent with that having the chord $A M$ as a basis are obtained. If the sum of the eight circular segments has a surface less than the value of which the area of the circle exceeds that triangle's, the surface $\Omega$ of the octagon FAMDGCHB is bigger than the surface of the triangle $E$. For, let us indicate the area of the eight 
circular segments with $S$. In the hypothesis of the theorem, such surface is less than the excess of which the area $C$ of the circle exceeds the area $E$ of the triangle. Namely $S<C-E$. However, it is $S=C-\Omega$. Thence, it holds $C-\Omega<C-E$, that is $\Omega>E$. Now, let us trace the perpendicular NO from the centre $N$ of the circle to the side $A F$ of the octagon. The segment $N O$ is less than the height of the triangle $E$, which (hypothesis) is equal to the radius. The perimeter of the octagon is smaller than the circumference, which is the other small side of $E$. You conclude that $\Omega<E$. This is in contradiction with the just obtained result. This absurdity implies that the surface of the circle cannot be bigger than $E$.

In a similar manner, it is possible to prove that the surface of the circle cannot be smaller than $E$, so that, it is equal.

This demonstration induces a series of conceptual questions, which are very formative in an educative context. Thence, the following didactical considerations can be developed in the classroom after having expounded Archimedes' proof:

1. In the previous proof there is a sensible point: Archimedes seems to give for granted that the perimeter of a polygon inscribed in a circumference is smaller than the circumference. This is intuitive. But the teacher should explain that intuition is a good guide from a heuristic point of view, but it might be very deluding if one relies only on intuition. Many mistakes might be possible. Archimedes was an extremely refined logic and he had grasped the problem. Thence, after having understood that, within the picture offered by Greek geometry, the proof that the straight line is the shortest path to connect two points was anything but trivial, he posed axiomatically that the smallest of all the lines having the same extremes is exactly the straight line (On the Sphere and Cylinder).

2. There is a further problem: in the expounded proof, it might happen that the difference between the area of the circle and that of the regular polygons inscribed within the circumference tends to diminish while the number of the polygons' sides increasing, but that it has an asymptotic value different from 0 . Archimedes was perfectly aware of this question and solved it in the first six propositions of On the Sphere and Cylinder. For, he showed that it is always possible to inscribe in a circumference a series of polygons whose area gets any ratio or difference with the circle's area. He also proved the same for the circumscribed polygons. These considerations might be connected by the teacher to the meaning and the use of the so called "Archimedes axiom".

3. The following consideration is fundamental in an educative perspective: among the historians of mathematics there are various ideas with regard to the relation between Archimedes' exhaustion method and integral calculus: there are historians who think that Archimedes was very close to discover integral calculus and other who, instead, think that there are scarce relations between his method and integral calculus, that Archimedes did not have a uniform method to calculate areas and volumes and that the relations between his exhaustion procedure and integral calculus are extrinsic, at all. In our context, this problem has only to be faced in a didactical and methodological perspective, not in a historical one.

Intuitively and not precisely, but to summarize: when the integral of a function of one real variable is calculated, the surface under the graphic of the function is divided into two series of (potentially) infinitesimal elements which can be considered rectangular without appreciable mistakes. One series approximates the required area by excess and the other series by defect. Under appropriate and specifiable conditions, the two series converge to the same sum, so that the required area under the function converges to the same value, as well. Analogously, Archimedes finds "infinitely small" elements of areas, whose sum is smaller than a pre-assigned area if the polygons are inscribed in the circle, case of those inscribed if they are circumscribed (we have analysed only the case of those inscribed). This is conspicuous in the presented proof and things work in a similar manner for any argument based on the exhaustion method. Therefore, the sum of infinitely small areas (obviously the locution "infinitely small" indicates a potential infinity) is a strong element shared by integral calculus and by Archimedes' method of exhaustion. Here the fundamental concept of mathematical analysis in involved: the concept of limit. There is no doubt that it is at the basis of integral calculus. But what to say with regard to Archimedes? It is possible to claim that he had the idea of a variable magnitude which approximates a given magnitude for a difference which is less than any pre-assigned magnitude. In this sense, he had the concept of limit. However, this is the first step towards the concept of limit, the second step consists in the idea that, exactly because the difference (an analogous reasoning can be made with the ratio) of two magnitudes becomes smaller than any pre-assigned magnitude, then, at limit, such magnitudes are equal. This idea is clearly expressed by Newton in his method of first and last ratios. There are not elements to claim if this was Archimedes' idea, too. On the other hand, independently of having conceived such idea Archimedes - and the entire Greek mathematics - did not have the algebraic means to develop the calculations of the limits. For example, in the specific case, how could Archimedes calculate the limit of the surface of the inscribed regular polygons? How could he be sure that their perimeter tends to the 
ISSN 1648-3898/Print/

ISSN 2538-7138/Online/

circumference and that their apothem tends to the radius? Let us remember that he proved that the difference between the surface of the circle and that of the inscribed polygons tends to 0 , but not that the surface of the inscribed polygons, when the number of their sides tends to infinity, tends to the triangle $E$. Archimedes avoided all these problems by resorting to the described ad absurdum reasoning. Thence: 1) Archimedes had some precise ideas which will converge to the concept of limit in modern sense; 2 ) it is uncertain whether he guessed the full theoretical features of this concept; 3 ) it is certain that he could not develop a calculation with the limits, given the restricted knowledge of algebra typical of Greek mathematics.

These considerations are very formative in the perspective of science and mathematics education because they accustom the learners to reason on the subtle nuances of the mathematical concepts, on their tenuous, but fundamental differences and on the logic behind any mathematical method.

4. Finally, in several of its applications, the exhaustion method runs like this: there are two figures $A$ and $B$ of which the identity of their areas or volumes has to be proven. Be known the area or volume of $B$. Suppose that $A \neq B$. It is possible to prove that an infinite series of other quantities $Q_{n}$, which are regularly constructible, exists such that, from a certain index $i$, it is $\left|A-Q_{i}\right|<\varepsilon$, being $\varepsilon$ an arbitrarily small quantity. Given this premise, the development of the reasoning concludes that the quantity $Q_{i}$ would be both smaller and bigger than $B$. This is an absurdity deriving from the hypothesis that $A \neq B$. Thence, this hypothesis has to be removed and $A=B$. As a matter of fact, there are different applications of the exhaustion method. The great historian of mathematics Dijksterhuis (see his Archimedes. Princeton: Princeton university Press, 1987 [original edition 1938], pp. 130-133) distinguishes two applications: the compression method, divided into two cases: when either a ratio or a difference of magnitudes is considered and the approximation method. It is not possible enters the details, but it is possible to point out that the here presented exhaustion method is the approximation variant. In any case it is not allowed to know a priori how the compressing figures or the approximating figures have to be constructed. Thence, some historians of mathematics conclude that the exhaustion method is not, in fact, a true method, but only a procedural idea. This can introduce an interesting discussion to develop in the classroom with regard to the concept of mathematical method. The teacher should explain that, if by "method" you mean a sort of mathematical machine which, given some data in entrance offers the result in exit by mechanical procedures, then the exhaustion method is not a method, but also the integral method is mechanically applicable only to some (restricted) well specifiable classes of functions. It is, however, certainly true that the integral method is more general than the exhaustion one. Furthermore, by an integral you can calculate the area or the volume of a figure without having any idea a priori with regard to the value of such area or volume. Namely, the integral method is also a heuristic procedure, whereas you can use the exhaustion method only if you have already an idea of the result you are going to obtain. Exhaustion is - as any ad absurdum reasoning - a demonstrative procedure, not a heuristic one. This granted, it is however undisputable that all the proofs by exhaustion share many common elements and that you know that a series of compressing or approximating figures have to be found, which is a very precise idea. Therefore, under this respect exhaustion is a method. On the hand, you do not know a priori what such figures should be. This is the inventive and creative part of the method, the one which is very formative in the context of mathematics education.

Therefore, the teacher should give the learners the general features of the exhaustion, should invite them to reflect on how to apply the method to different cases, starting from some easy example arriving to more complex ones. This will contribute to develop both the creativity and the fantasy of the pupils as well as their logical skills, which is an amazingly important result for any educative process. After some lessons framed in this manner, the teacher should explain the limitations to which exhaustion method is subject and should formalise the introduction of integral method. But now the need of such a formalization will be completely clear for the pupils and will arrive after a very formative training of which they have been active protagonist and not passive spectators. 